\title{
A new approach to laser polishing and form correction of optical components
}

\author{
Harold Kessler $^{1, *}$, Roelene Botha ${ }^{1}$, and Carsten Ziolek ${ }^{1}$ \\ ${ }^{1}$ NTB Interstaatliche Hochschule für Technik Buchs, Institute for Production metrology, Materials and Optics, 9471 Buchs SG, \\ Switzerland
}

\begin{abstract}
The increasing variety of optical components and materials, combined with stricter surface tolerance requirements, necessitate refining existing polishing processes and developing innovative new polishing solutions and metrology technologies. A fast, reproducible laser polishing process would offer considerable economic benefits over conventional mechanical polishing processes and interest a broad variety of optics manufacturers. In this work, a holistic approach is taken to address the various aspects of glass polishing and form correction via a novel laser polishing system design, the use of a measurement strategy that can be integrated inline and simulation results that are correlated with process parameter studies for different materials.
\end{abstract}

\section{Introduction}

The use of aspheres have been attracting a large amount of attention as they enable lens system designers to decrease the number of lenses, and consequently the number of optical surfaces, used. This lowered complexity can improve the performance of devices, reduce waste and lower the manufacturing cost. Achieving surface figure and roughness values for such freeform optics that are comparable with those of standard precision optical components still remains elusive and requires a full understanding of the complete production technology chain. This is not straightforward, when considering the complexity of the current optical component fabrication process. Typical manufacturing steps include shaping, grinding and edge grind and polish processes, followed by iterative intermediate, final and small tool (figure correcting) polishing steps that include intermittent ex-situ surface characterization measurements. The above described conventional methods for polishing optics can rightly be considered as «more an art than a science». [1] The variety of process parameters affect the final output of the process, but their individual and mutual effect is not clear. [2] It is of interest to have a stable and fully understood process independent of material or product shape.

\subsection{Laser polishing of glass: State-of-the-art}

The main advantage of laser polishing is that it allows avoiding the restrictions of conventional mechanical polishing methods and their tools. Furthermore, polishing errors, such as sub-surface damage, can be completely avoided by using laser polishing.
Laser polishing of fused silica surfaces was first done to increase the damage resistance against laser radiation of shorter wavelengths. [3] Efficient polishing processes of fused silica surfaces down to few nanometres have been shown by various research teams. [4-6] Hildebrand et al.[4] performed a numerical simulation describing the influence of several process parameters. To monitor the laser polishing and shape correction process, a pyrometer or spectrometer can be used to continuously measure the temperature in the polishing spot and accordingly adjust the laser power $[7,8]$. The process can also be visualized using imaging techniques based camera systems or laser beams and zoom-telescopes $[9,10]$. By monitoring the actual laser power using a photo diode in the laser beam delivery system, the laser power can be corrected as required using a proportional-integral-derivative (PID) feedback controller [11]. Many crucial aspect of the laser polishing process, process control and necessary metrology are still not fully understood and many open questions remain.

\section{Approach}

\subsection{Considerations for the laser polishing strategy}

This work aims to establish a fully understood, reliable and automated laser polishing process to produce freeform optics with surface figure and roughness values comparable with those of precision optical components. A simulation considering additional temperature dependent effects to describe the finished surface morphology is also developed. The following points are taken into consideration for the development:

- Selection of the polishing strategy.

* Corresponding author: harold.kessler@ntb.ch 
The polishing strategy between different research teams is strongly varying. Some use fixed parameters while others regulate their process with feedback loops. The impact of different polishing strategies on the surface, as well as trade-off between expense and achieved result will be analysed.

- Increasing the variety of materials that can be processed.

Fused silica is the material of choice for laser polishing, especially with $\mathrm{CO}_{2}$ laser polishing. This is due to the ideal absorption characteristics of fused silica in this wavelength regime. Weingarten et al. [6] further successfully polished BK7 and S TIH6 in their work.

- Correlating the simulation results with the observed material changes.

Being able to simulate the process for different parameters and materials is desirable. An approach for the simulation was already done by Hildebrand et al. [4]. This model is analyzed and further extended with additional parameters.

- Developing a surface measurement strategy that can be implemented in-line.

In-situ measurement of the samples during the polishing process can be used to further reduce the time needed compared to conventional polishing.

- High temperature stability of the polished components.

Some of the physical effects utilised for laser polishing, e.g. for the form correction after polishing can be reversed with annealing. This effect has to be taken into account.

\subsection{In-line measurement strategy}

The measurement of the roughness and form of the optical component needs to adapt to the component's shape. As free-form optics are becoming increasingly important, this constitutes a serious obstacle. Optical measuring methods such as chromatic confocal microscopy, laser scanning microscopy, Phase Measuring Deflectometry (PMD), white light interferometry and optical coherence microscopy, as well as a combination between an optical measuring technology and AFM, may offer the required flexibility and resolution. Furthermore, acquisition of measurement data in real-time could also be used to improve the stability of the polishing process.

In the PMD method [12] shown in Fig. 1, twodimensional sinusoidal fringes are projected onto the surface. Varying distances between the projector and the surface will result in an observable change of the fringes. This variation is recorded by a camera. The fringes are phase shifted and another picture is taken. After several iterations, the surface profile and curvature can be deduced. PMD is a rather cost effective and robust way to measure 3D freeform surfaces, especially for specular reflection surfaces.

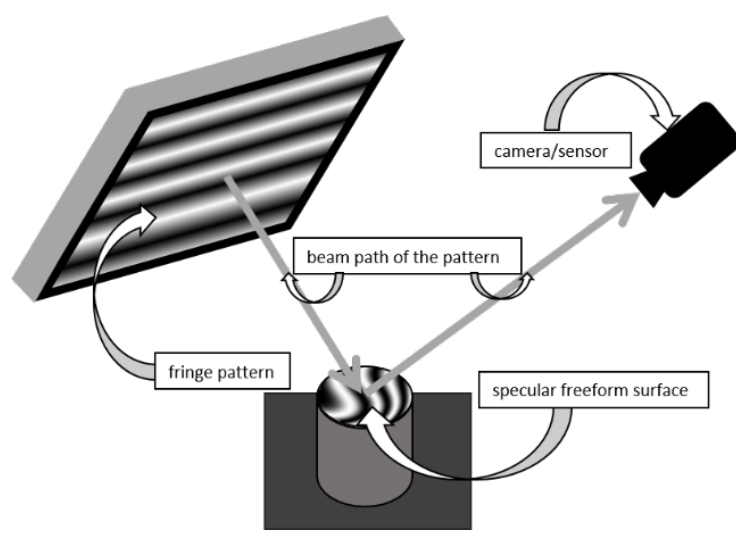

Fig. 1. Schematic of the PMD setup.

\section{Conclusion}

This work discussed the considerations that were taken into account for developing a new laser polishing process for optical freeforms. A concept for a $\mathrm{CO}_{2}$ laser polishing system was discussed and a surface measurement strategy that can be implemented in-line suggested. We have started studying the process windows for fused silica and sapphire. In a consequent step, the results of the parameter study will be compared with simulation results. A comparison of the influence different polishing strategies have on the efficiency and the stability of the polishing process will then be performed.

\section{References}

1. I. D. Marinescu, E. Uhlmann, T. Doi, Handbook of Lapping and Polishing(CRC Press, 2006)

2. D.K. Mandal, C. S. Syan, CAD/CAM, Robotics and Factories of the Future)

3. P.A. Temple, W. H. Lowdermilk, D. Milam, Appl. Opt. 12 (1982)

4. J. Hildebrand, K. Hecht, J. Bliedtner, H. Müller, Physic Procedia, 39, 277-285 (2012)

5. M. J. Matthews, S. T. Yang, N. Shen, S. Elhadj, R. N. Raman, G. Guss, I. L. Bass, M. C. Mostrand, P. J. Wegner, Adv. Eng. Materials 17, 247-252 (2015)

6. C. Weingarten, A. Schmickler, E. Willenborg, K. Wissenbach, R. Poprawe, J. Laser Apps. 29 (2017)

7. S. Heidrich, A. Richmann, P. Schmitz, E. Willenborg, K. Wissenbach, P. Loosen, R. Poprawe, Optics and lasers in Engineering 59, 34-40 (2014)

8. B. Burzic, M. Hofele, S. Mürdter, H. Riegel, J. of Laser Applications, 29, 011701 (2017)

9. O. Pütsch, A. Temmler, J. Stollenwerk, E. Willenborg, P. Loosen, Proc. SPIE 8843 (2013)

10. A. Temmler, E. Willenborg, K. Wissenbach, Proc. SPIE 8243, 82430W (2012)

11. E.V. Bordatchev, 3rd LAP Conf, Aachen, DE (2018)

12. F. Z. Fang, X. D. Zhang, A. Weckenmann, G. X. Zhang, and C. Evans, CIRP Annals, 62 823-846 (2013) 